\title{
Rapidly Progressive Malignant Fibrous Histiocytoma of Right Atrium: a Rare Case Report
}

\author{
Timucin Aksu', MD; Safa Gode1, MD; Kursat Oz ${ }^{1}$, MD; Burak Ersoy ${ }^{1}$, MD; Cigdem Tel Ustunısık , MD; Yesim Guner ${ }^{1}$, \\ MD; Omer Faruk Atay ${ }^{2}$, MD; Vedat Erentug ${ }^{1}, \mathrm{MD}$
}

DOI: 10.21470/1678-9741-2018-0114

\begin{abstract}
We are going to present a case of malignant fibrous histiocytoma in the right atrium, which is a very rare entity. The patient had a right atrial mass, which prolapsed through the tricuspid valve into the right ventricle, causing functional tricuspid valve stenosis. The tumor was completely resected and the patient had an uneventful postoperative period. Histopathological examination reported malignant fibrous histiocytoma. The patient presented to the emergency department five weeks after discharge with dyspnea and palpitation. Echocardiography and magnetic resonance imaging
\end{abstract}

revealed recurrent right atrial tumor mass. His clinical status has worsened, with syncope and acute renal failure. On the repeated echocardiography, suspected tumor recurrence was observed in left atrium, which probably caused systemic embolization. Considering the aggressive nature of the tumor and systemic involvement, our Heart Council decided to provide palliative treatment by nonsurgical management. His status deteriorated for the next few days and the patient succumbed to a cardiac arrest on the $4^{\text {th }}$ day.

Keywords: Undifferentiated Pleomorphic Sarcoma. Malignant Fibrous Histiocytoma. Right Atrium.

\begin{tabular}{ll}
\hline Abbreviations, acronyms \& symbols \\
\hline CPB & $=$ Cardiopulmonary bypass \\
CT & $=$ Computed tomographic \\
ECG & $=$ Electrocardiogram \\
LAD & $=$ Mid-left anterior descendant \\
MFH & $=$ Malignant fibrous histiocytoma \\
MRI & $=$ Magnetic resonance imaging \\
PD & $=$ Posterior descendant \\
TEE & $=$ Transesophageal echocardiography \\
TTE & $=$ Transthoracic echocardiography \\
UPS & $=$ Undifferentiated pleomorphic sarcoma \\
\hline
\end{tabular}

\section{INTRODUCTION}

Undifferentiated pleomorphic sarcoma (UPS), also known as malignant fibrous histiocytoma (MFH), is excessively rare among primary cardiac tumors ${ }^{[1]}$. The average survival time of patients with MFH is defined as short as only one year in the literature ${ }^{[2]}$. Therefore we are going to present a case with rapidly progressive MFH of the right atrium.

'Department of Cardiovascular Surgery, Istanbul Mehmet Akif Ersoy Thoracic and Cardiovascular Surgery Training and Research Hospital, İstanbul, Turkey.

${ }^{2}$ Department of Pathology, Kanuni Sultan Suleyman Training and Research Hospital, İstanbul, Turkey.

This study was carried out at the Department of Cardiovascular Surgery, Istanbul Mehmet Akif Ersoy Thoracic and Cardiovascular Surgery Training and Research Hospital, İstanbul, Turkey.

\section{CASE REPORT}

A 56-year-old male patient was admitted to our clinic with dyspnea and general fatigue for two months. Physical examination revealed stable hemodynamics with a 2/6 diastolic murmur in the tricuspid area. The patient was tachypneic, with crepitations in bilateral lung bases. Clinical examination of the abdomen was normal and there was no peripheral edema.

Laboratory investigations including cardiac markers, hemoglobin, hematocrit, urea, creatinine, and C-reactive protein levels were normal. Electrocardiogram (ECG) and chest $x$-ray were essentially normal. Transthoracic echocardiography (TTE) revealed normal aortic and mitral valves and normal ventricular functions. Besides, a multi-lobed, heterogeneous, and $45 \times$ $24 \mathrm{~mm}$ mass, originating from the anterior leaflet of tricuspid valve, was observed in right atrium. It prolapsed through the tricuspid valve into the right ventricle during diastole, leading to functional tricuspid stenosis. The tumor was verified by transesophageal echocardiography (TEE). Contrary to TTE, TEE revealed a $47 \times 30 \mathrm{~mm}$ mass in the right atrium. No other mass was detected and no other mass in cranium, thorax, abdomen, and pelvis in 640-slice computed tomographic (CT) scan (Figure 1A) was observed. Cardiac magnetic resonance imaging (MRI)

Correspondence Address:

Safa Gode

iD https://orcid.org/0000-0002-5505-1783

Mehmet Akif Ersoy Thoracic and Cardiovascular Training and Research Hospital Department of Cardiovascular Surgery

İstasyon Mah.Turgut Özal Bulvarı No:11 34000 - Küçükçekmece, Istanbul, Turkey

Zip Code: 3400

E-mail:safagode@yahoo.com 
revealed an approximately $35 \mathrm{~mm}$ lobulated hypointense mass lesion, which prolapsed from the right atrium into the right ventricle (Figure 1B). Also, coronary angiography showed $70 \%$ of the stenosis in the mid-left anterior descendant (LAD) artery and $90 \%$ of the stenosis in the proximal posterior descendant (PD) artery (Figure 1C). We decided to proceed with tumor excision simultaneously with coronary artery bypass grafting.

Median sternotomy was performed, and standard cardiopulmonary bypass (CPB) was carried out with aortic and two-stage venous cannulation. Right atrium was opened and a $43 \times 33$ mm lobulated mass lesion was observed on the orifice of the tricuspid valve. Tricuspid intervention was deferred since the tricuspid valve was normal, without any tumoural attachment. The tumor's pedicle was on the interatrial septum (Figure 2A).
Therefore, it was almost completely excised together with interatrial septal tissue (Figure 2B). No tumor was observed in the transseptal inspection of the left atrial cavity. After closure of the atriums, LAD and PD arteries were anastomosed with saphena vein grafts. Then, cross clamp was removed and proximal anastomosis of saphena vein grafts were performed. CPB was gradually weaned. There was no residual tumor in perioperative TEE. Pathological examination revealed the diagnosis of MFH (Figures 2C and D). Postoperative course was uneventful. Therefore the patient was discharged on postoperative day seven.

On the fifth week of discharge, the patient was readmitted to our emergency clinic with severe dyspnea (oxygen saturation: 88\%) and palpitation (heart rate: 135 pmn). Cardiac markers and
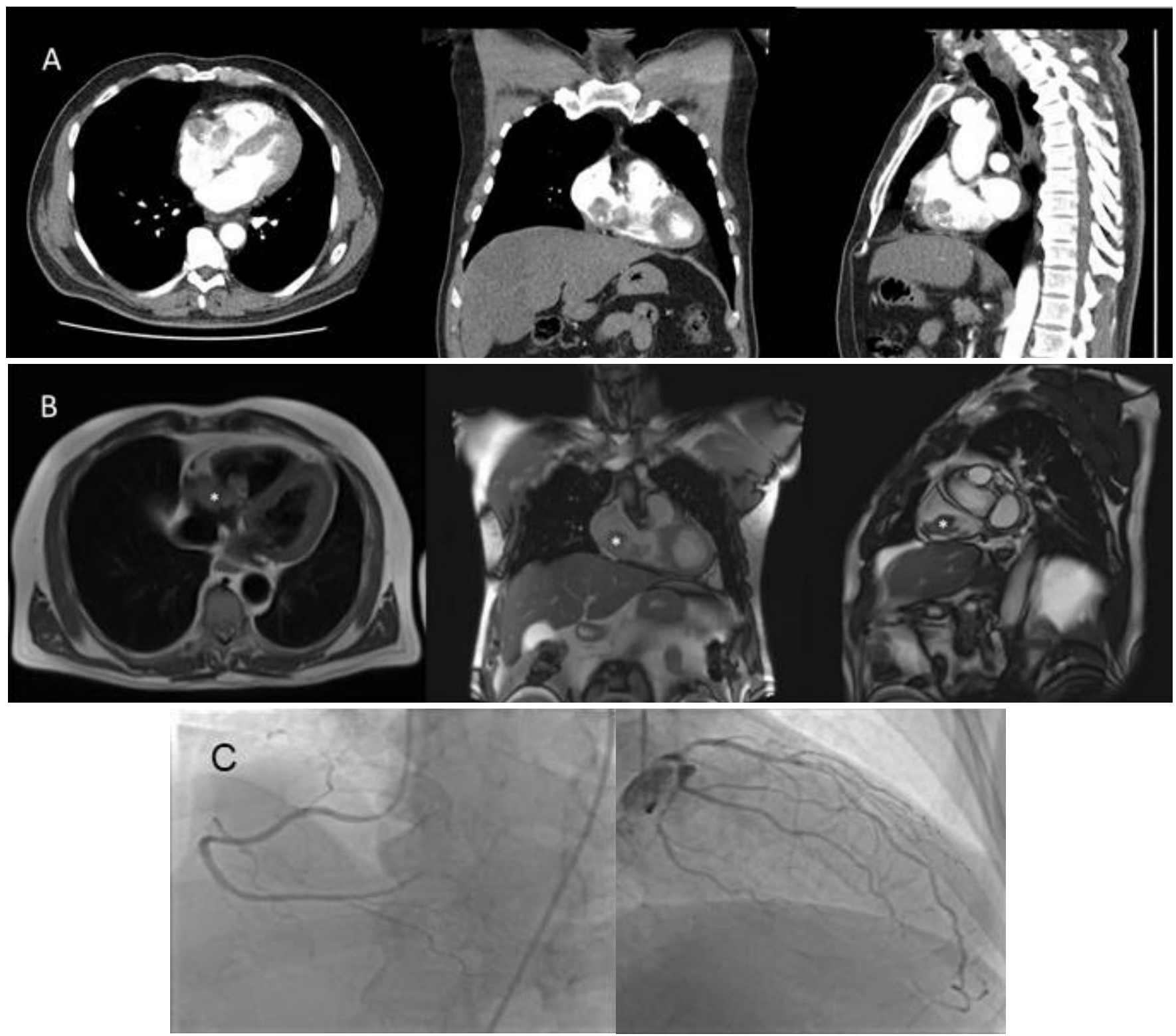

Fig. 1 - A) Computed tomographic image. B) Magnetic resonance imaging showing mass in the right atrium. C) Coronary angiography showing stenosis in mid-left anterior descendant and posterior descendant arteries. Tricuspid Valve. 

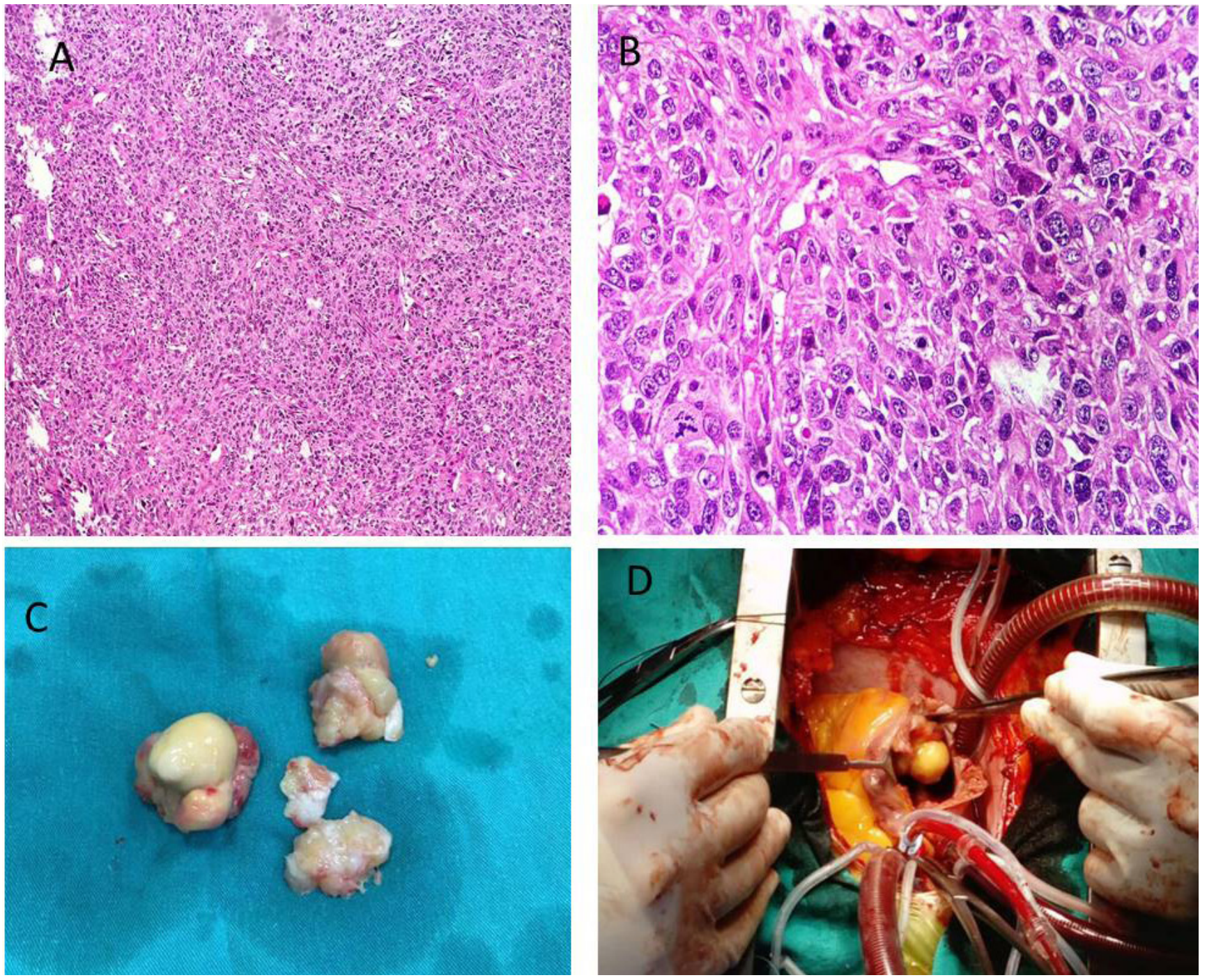

Fig. 2 - A) Surgical view of mass in the right side of the right atrium. B) Excised mass. C) Hematoxylin-eosin stain showing malignant fibrous histiocytic cells (magnification 4). D) Magnification 40. There was mass attached to the anterior leaflet of the tricuspid valve.

ECG were normal in terms of ischemia. Hemoglobin, hematocrit, C-reactive protein, urea, and creatinine levels were $10.2 \mathrm{~g} / \mathrm{dl}$, 31\%, $368 \mathrm{mg} / \mathrm{dl}, 96 \mathrm{mg} / \mathrm{dl}$, and $3.15 \mathrm{mg} / \mathrm{dl}$, respectively. Other laboratory parameters were normal. TTE revealed a recurrent mass of $32 \times 23 \mathrm{~mm}$ that led to stenosis of tricuspid valve. There weren't any disorders in mitral and aortic valves. In cardiac MRI, a $36 \times 22 \mathrm{~mm}$ bilobulated mass was detected in the right atrium (Figure 3). Although there was no evidence of tumor presence on the left side of the heart in MRI, TEE revealed a suspicious tumor image in the left atrium. The patient experienced a syncope episode on the following day. On the same day, hemodiafiltration was initiated due to acute decompensated renal failure. The case was discussed by our Heart Council and it was decided for nonsurgical palliative treatment because of the very high grade tumor and the patient's unconsciousness. His status deteriorated over the next few days and the patient succumbed to a cardiac arrest on the 4th day of readmission.

\section{DISCUSSION}

Recently, the name MFH was converted to UPS, as the tumor is formed by atypical fibroblastic cells, with dispersed collagen, and has the classic herringbone pattern microscopically. In our patient, this tumor was diagnosed as MFH with vascular and smooth muscle differentiation. Less than 100 patients with primary MFH have been reported in the literature ${ }^{[3]}$. In addition to that, primary cardiac MFH is diagnosed even more rarely and it affects patients with a wide range of age: 14 to 77 years ${ }^{[4]}$. The tumor is mostly seen in the left atrium, with a frequency of $65 \%{ }^{[3]}$. Contrarily, in our case, the tumor was in the right atrium in the first admission. Although usually these patients present to hospital with cardiac symptoms, including arrhythmias, valve dysfunction, or heart failure, the symptoms, in our case, were dyspnea and general fatigue. These symptoms were described with various mechanisms by Shanmugam et al. ${ }^{[5]}$. 


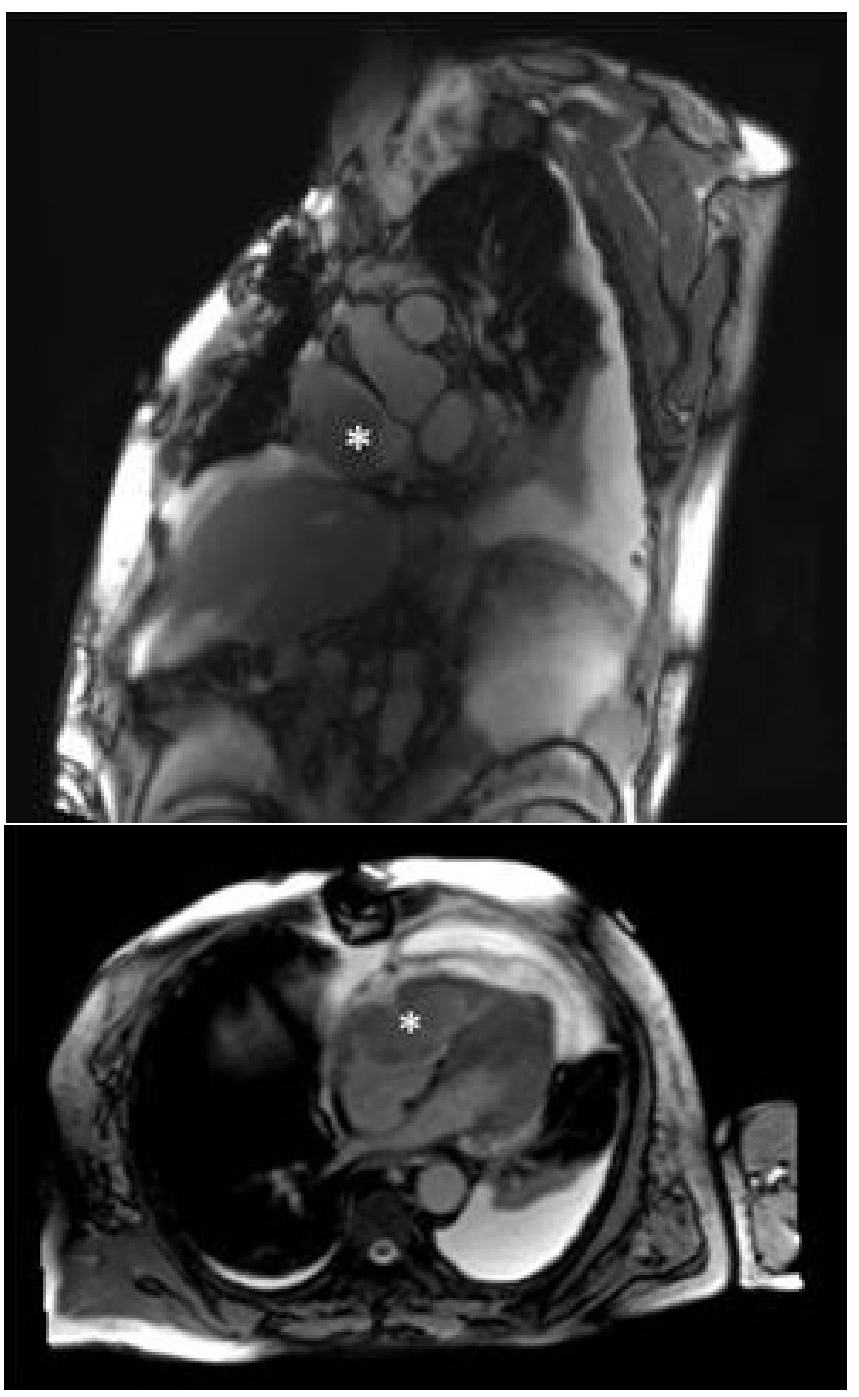

Fig. 3 - Magnetic resonance imaging showing recurred mass in the right atrium.

* $=$ Right atrial mass.

Cardiac MRI is used for differential diagnosis in cardiac mass, and high-resolution $C T$ is used easily and is widely applied for diagnosis. Also, TTE is an excellent method for diagnosis of cardiac mass; it shows the tumor's location and evaluate the valves' function and the hemodynamic effect of tumor in the heart.

The treatment strategy for MFH depends on the tumor's size, location, histological formation, and metastatic status. The ideal surgical procedure for eligible patients with cardiac MFH without metastasis is a complete resection of the tumor. However, MFH invades the heart tissue deeply and generally affects whole cardiac structures. Therefore, complete surgical resection is very difficult and mortal, because of the postsurgical progress. In our case, the tumor that caused tricuspid stenosis was in the right atrium and its pedicle was on the interatrial septum. However, after tumor resection, the tricuspid valve was normal in perioperative TEE. Despite almost complete resection of tumor in the presented case, a very quick recurrence has developed in about five weeks.
In second admission, the patient's symptoms could be related to tricuspid stenosis due to mass. In the readmission period, despite the absence of tumor in the left atrium in cardiac MRI, tumor recurrence was suspected on the left side in TEE, which may cause systemic embolism. Because of the loss of consciousness and acute renal failure, there could be tumor embolism to cerebral and renal arteries. Additionally, cardiac arrest could occur due to coronary artery embolism. This fragile tumor on the right side of the heart could also cause pulmonary embolism. Therefore, dyspnea and palpitation could develop due to pulmonary embolism in readmission period. Thereby, nonsurgical palliative treatment was preferred by our Heart Team. Average survival of patients with sarcomas in the right side of the heart is only six months. Despite applying radiotherapy or chemotherapy, patients with cardiac sarcoma without surgical resection typically live about nine to 12 months $^{[6]}$. Consequently, the benefit of chemotherapy and radiotherapy in primary cardiac sarcomas is unclear. And because of the patient's unconsciousness and poor clinical status, chemotherapy or radiotherapy were also not suggested by oncology in our case. In conclusion, like in our case, cardiac MFH is a very rare tumor with difficult surgical complete resection, fast progression, and poor survival.

\section{No financial support.}

No conflict of interest.

\section{Authors' roles \& responsibilities}

TA Drafting the work or revising it critically for important intellectual content; final approval of the version to be published

SG

Substantial contributions to the conception or design of the work; or the acquisition, analysis, or interpretation of data for the work; final approval of the version to be published

KO Agreement to be accountable for all aspects of the work in ensuring that questions related to the accuracy or integrity of any part of the work are appropriately investigated and resolved; final approval of the version to be published

BE Agreement to be accountable for all aspects of the work in ensuring that questions related to the accuracy or integrity of any part of the work are appropriately investigated and resolved; final approval of the version to be published

CTU Drafting the work or revising it critically for important intellectual content; final approval of the version to be published

YG Substantial contributions to the conception or design of the work; or the acquisition, analysis, or interpretation of data for the work; final approval of the version to be published

OFA Agreement to be accountable for all aspects of the work in ensuring that questions related to the accuracy or integrity of any part of the work are appropriately investigated and resolved; final approval of the version to be published

VE Final approval of the version to be published 


\section{REFERENCES}

1. Fletcher CDM, Chibon F,Mertens F. Undifferentiated/unclassified sarcomas. In: Fletcher CDM et al, editor. WHO classification of tumours of soft tissue and bone. 4th ed. Lyon: IARC; 2013. p. 236-8.

2. Burke AP, Cowan D, Virmani R. Primary sarcoma of the heart. Cancer. 1992;69(2):387-95.

3. Bandyopadhyay S, Banerjee S, Paul A, Das RK. Primary malignant fibrous histiocytoma involving the left pulmonary vein presenting as a left atrial tumor. Ann Card Anaesth. 2013;16(4):293-5. doi:10.4103/0971-9784.119184.
4. Okamoto K, Kato S, Katsuki S, Wada Y, Toyozumi Y, Morimatsu M, et al. Malignant fibrous histiocytoma of the heart: case report and review of 46 cases in the literature. Intern Med. 2001;40(12):1222-6. doi:10.2169/ internalmedicine.40.1222.

5. Shanmugam G. Primary cardiac sarcoma. Eur J Cardiothorac Surg. 2006;29(6):925-32. doi:10.1016/j.ejcts.2006.03.034.

6. Schena S, Caniglia A, Agnino A, Caruso G, Ferlan G. Survival following treatment of a cardiac malignant fibrous histiocytoma. Chest. 2000;118(1):271-3. doi:10.1378/chest.118.1.271. 